\title{
Análisis comparativo entre el doctorado profesional y de investigación en México
}

\author{
Josefina Patiño Salceda
}

\section{RESUMEN}

Se analiza la evaluación de los doctorados en el Programa Nacional de Posgrados de Calidad (PNPC) para identificar bajo qué criterios se justifica el doctorado profesional, a través de su comparación con el doctorado de investigación. A partir de la revisión documental se identifican dos tendencias sobre la definición de la educación doctoral: una orientada hacia la armonización y otra hacia la diversificación de doctorados. El PNPC se ubica en esta última tendencia, al proponer un doctorado profesional que se distingue del de investigación por valorar el aprendizaje adquirido tanto en la academia como en el trabajo; de esta manera se impulsa la investigación aplicada para el desarrollo de las profesiones y la vinculación de los graduados con el sector productivo.

Palabras clave: doctorado, evaluación de programas, formación de investigadores, política científica, profesiones, México.

\section{Josefina Patiño Salceda}

Mexicana. Doctora en Educación por la Universidad Autónoma del Estado de Morelos (UAEM), México. Becaria del Instituto de Investigaciones sobre la Universidad y la Educación, Universidad Nacional Autónoma de México (UNAM) [asesorada por el Dr. Alejandro Márquez Jiménez]. Profesora en la Universidad Pedagógica Nacional, Unidad 096, México. Temas de investigación: políticas y procesos de formación del posgrado en México. 


\section{Análise comparativa entre o doutorado profissional e de pesquisa no México}

\section{RESUMO}

Se analisa a avaliação dos doutorados no Programa Nacional de Pós-graduação de Qualidade (PNPC - por sua sigla em espanhol) para identificar sob que critérios se justifica o doutorado profissional, através da comparação com o doutorado de pesquisa. A partir da revisão documental se identificam duas tendências sobre a definição da educação doutoral: uma orientada para a harmonização e outra para a diversificação de doutorados. O PNPC se localiza nesta última tendência, ao propor um doutorado profissional que se distingue do de pesquisa por valorizar a aprendizagem adquirida tanto na universidade como no trabalho; desta maneira se impulsa a pesquisa aplicada para o desenvolvimento das profissões e a vinculação dos formandos com o setor produtivo.

Palavras chave: doutorado, avaliação de programas, formação de pesquisadores, política científica, profissões, México.

\section{Comparative Analysis Between the Professional and Research Doctoral Degrees in Mexico}

\section{ABSTRACT}

The author analyzes the assessment of doctorate programs within the framework of the National Quality Postgraduate Program (PNPC) in order to identify under which criteria the professional doctoral degree is justified by means of a comparison with the research PhD. Two trends in the definition of doctoral education are identified from the documentary review: one oriented towards harmonization and the other towards the diversification of doctorates. The PNPG has chosen to stick to this last trend, by proposing a professional doctorate that differs from the research $\mathrm{PhD}$ by valuing the learning acquired both in academia and in the workplace; in this way, applied research is promoted for the development of professions and the link between graduates and the productive sector.

Key words: doctorate, evaluation of programs, training of researchers, scientific policy, professions, Mexico. 


\section{Introducción}

Los doctorados profesionales, tal como se conocen en estos días, se identificaron durante el siglo $\mathrm{XX}^{1}$, como propuesta alterna al doctorado de investigación; su aparición, sin embargo, ha sido polémica y ha carecido de aceptación en algunos espacios académicos (Schildkraut y Stafford, 2015; Bao Kehm y Ma, 2016). Esto explica, en parte, un interés relativamente tardío para apoyar su inserción en los formatos de evaluación de la calidad, y para abordarlo como objeto de estudio dentro del campo de la investigación educativa.

En el caso de México, el doctorado profesional se incorporó al Programa Nacional de Posgrados de Calidad (PNPC) ${ }^{2}$ en 2014, pero no se ha discutido el significado y los elementos que integran esta propuesta; así, el objetivo de este artículo ${ }^{3}$ es analizar el instrumento de evaluación del PNPC para identificar bajo qué criterios se justifica el doctorado profesional en comparación con el de investigación.

Se utilizó un método descriptivo-comparativo que permite "hacer comprensibles las cosas desconocidas a partir de cosas conocidas" (Grosser, en Nohlen, 2006: 2). Este método es ideal debido a que el doctorado profesional ha sido adoptado para convertirse en una opción alterna al doctorado de investigación. El análisis consistió en la descripción y comparación de las orientaciones de investigación y profesional, a través de sus principales rasgos, descritos en el Marco de Referencia para la Evaluación y Seguimiento de Programas de Posgrado Presenciales (CONACyT, 2015a), versión 6. Asimismo, se analizaron los criterios de evaluación para cada orientación y se compararon sólo aquellos en los que se encontraron diferencias. Para esto se analizaron el Anexo A: Programas de Orientación a la Investigación, versión
4.1 (2015b); y Anexo A: Programas de Orientación Profesional (2015c), versión 4.1. De esta manera fue posible identificar los aspectos que distinguen a ambos tipos de doctorado.

En este artículo también se exponen los resultados de la revisión documental que se llevó a cabo sobre las definiciones de doctorado establecidas por las agencias de evaluación y acreditación de la calidad. Dado que el tema del doctorado profesional es emergente, se asumió que la presencia de definiciones de este doctorado en los instrumentos de evaluación podía ser inicial o inexistente; por ello, se recurrió al análisis de los nuevos tipos de doctorado a partir de acercamientos empíricos o documentales realizados por otros especialistas del tema.

En el artículo se desarrollan tres apartados: en el primero se exponen algunas críticas al doctorado de investigación; en el segundo apartado se describen dos tendencias sobre la definición y tipos de educación doctoral identificadas por la autora en distintas fuentes documentales como artículos de investigación, informes, marcos de referencia utilizados por agencias de evaluación, entre otros; y en el tercer apartado se presentan los resultados del artículo, basados en el análisis de los criterios de evaluación para los doctorados en el PNPG. Finalmente, se presentan las conclusiones.

\section{El destino laboral de los graduados de doctorado: principal motor para su transformación}

Una de las principales críticas hacia el doctorado tradicional es la insuficiencia de éste para formar investigadores con perfiles diversos para insertarse en tres ámbitos laborales: el académico, el gubernamental y el industrial. Para el caso de México, aunque se

\footnotetext{
${ }^{1}$ En los Estados Unidos de América aparecen a principios de 1920; en Inglaterra y Australia durante 1990 (Bourner et al., 2001; Taylor, 2004); en China, Islandia, Nueva Zelanda y Canadá durante la última década del siglo XX (Bao et al., 2016; Maxwell, 2003; Wildy et al., 2015).

${ }_{2}^{2}$ Principal instrumento de política que promueve la calidad del posgrado nacional; el Programa es administrado por el Consejo Nacional de Ciencia y Tecnología (CONACyT) y la Subsecretaría de Educación Superior de la Secretaría de Educación Pública (SEP).

${ }^{3}$ Este proyecto se elaboró con el apoyo del Programa de becas posdoctorales de la UNAM.
} 
carece de datos precisos sobre el principal destino laboral de los graduados, ${ }^{4}$ la percepción del director de Posgrados del CONACyT es que el principal empleador ha sido el sector académico (Patiño, 2017).

Este hecho no es específico de México; un estudio que compara el destino laboral de los graduados de doctorado por países (Auriol, 2010), muestra que la educación superior es el principal destino laboral de los graduados; en segundo lugar está el sector gubernamental, y en tercero, el sector empresarial. De 13 países participantes ${ }^{5}$ en el estudio, todos excepto uno, se ubican en un rango de 40 a $80 \%$ de graduados en el sector académico. Austria es el único país que toma distancia del esquema dominante al registrar una distribución más equilibrada entre sectores. Destaca el caso de Polonia, donde 80\% de los graduados se emplea en el ámbito académico, mientras que Austria, Bélgica y Estados Unidos de América (EUA), registran aproximadamente $30 \%$ de graduados en el sector empresarial, distinguiéndose como los tres países con mayor porcentaje en ese sector (Auriol, 2010).

¿Por qué existen estas diferencias entre los países? Teichler (2015) sugiere que el doctorado de investigación, basado en los principios de Humboldt, fue adaptado en diversos países de acuerdo con tres tipos de identidades académicas: la alemana, la anglosajona y la latinoamericana (Arimoto, en Teichler, 2015). ${ }^{6}$ Esto significa que las adaptaciones dependen de la interpretación que hacen las comunidades académicas y otros actores - a partir de sus propios referentes - sobre la idea original.
Las cifras relacionadas con los destinos laborales de los graduados de doctorado han propiciado que gestores y académicos lancen fuertes críticas al posgrado en general, y señalen su fracaso en la función de producir conocimiento innovador (Mollis y Jaim Etcherverry, en Mollis, 2010). Los autores de estas críticas consideran que el posgrado se ha limitado a la producción de conocimiento certificado por las propias élites, y que sólo se difunde a través de tesis y publicaciones académicas (Núñez, 2002). En el caso de México, el director del PNPG asegura que, del total de productos generados por los estudiantes de posgrado, solamente $4 \%$ se concreta en aplicaciones, y el resto deriva en publicaciones (Patiño, 2017). En síntesis, se considera que el posgrado se ha desarrollado bajo una visión académica, por lo que, no ha tenido un impacto social relevante (Morles y León, 2002).

Estas críticas se han agudizado - en el caso del doctorado - con la llegada del paradigma de la economía del conocimiento como referente ideal para el desarrollo económico y social. De acuerdo con políticas de corte internacional, la investigación, el desarrollo tecnológico y la innovación, son elementos clave para generar riqueza y bienestar. En el caso de México, la adopción de esta premisa ha sido evidente en los últimos tres documentos del Programa Especial de Ciencia, Tecnología e Innovación (PECiTI), principal documento sobre política científica en el país (Patiño, 2017). De esta manera, se espera que el doctorado satisfaga las necesidades de formación que requiere el paradigma mencionado.

\footnotetext{
${ }^{4}$ Con base en el diagnóstico del posgrado en México se sabe que, del total de egresados de posgrado, $33 \%$ labora en el sector académico; $31 \%$ en el sector gobierno, $29 \%$ en el sector empresarial y $7 \%$ en otros sectores (Bonilla, 2015); sin embargo, el estudio referido no presenta cálculos específicos que permitan distinguir niveles de posgrado. Dadas las enormes diferencias entre especialidad, maestría y doctorado, sería erróneo considerar estos datos como referencia para el doctorado.

${ }^{5}$ Los países son: Austria, Polonia, República Eslovaca, Rumania, Lituania, Estonia, Letonia, República Checa, Chipre, Estados Unidos de América, Bulgaria, España y Bélgica. El estudio incluyó personas que obtuvieron el grado de doctorado entre 1990 y 2006.

${ }^{6}$ En el caso alemán, la investigación es la clave de la identidad de los profesores universitarios; los jóvenes académicos reciben acompañamiento individual por parte de un "doctor padre" y la libertad académica es fundamental. En el caso anglosajón, los académicos valoran el vínculo estrecho entre investigación y enseñanza, y les otorgan un peso similar a ambos conceptos. En el caso latinoamericano, la docencia es el principal referente de la identidad de los profesores universitarios.
} 
Especialistas en el tema sugieren que el problema radica en la dificultad para definir la pertinencia de los programas; por ello se ha optado por llevar a cabo acciones en dos sentidos: una de ellas consiste en crear una perspectiva integradora cuyo objetivo sea: "profesionalizar la investigación y cientifizar/ tecnologizar la formación profesional", así como "Promover la complementariedad entre conocimiento científico y conocimiento aplicado" (Mollis, 2010: 47); la otra acción consiste en distinguir tres tipos de necesidades: a) las que corresponden a la propia disciplina de estudio, b) las del contexto económico y social y, c) las del desarrollo de las profesiones (Arias, en Valenzuela, 2014: 72). Así, el doctorado profesional se ubicaría en esta última postura.

\section{Tendencias en la educación doctoral: ¿hacia la armonización o la diversificación?}

La definición del doctorado encontrada en Europa, Latinoamérica, Estados Unidos, Australia y China permitió distinguir dos tendencias al respecto: a la primera se le denominó armonización de la educación doctoral, y a la segunda, diversificación de la educación doctoral.

Armonización de la educación doctoral. Esta tendencia agrupa todas aquellas definiciones del doctorado que no distinguen tipos ni orientaciones. No obstante, se pueden identificar dos definiciones: una tradicional, que adoptó aspectos de la identidad académica alemana de Humboldt, y otra definición renovada, que adopta exigencias como la interdisciplinaridad en la investigación, la pertinencia, la innovación, la internacionalización, etcétera.

Diversificación de la educación doctoral. Esta tendencia se caracteriza por reunir una diversidad de tipos de doctorado como el profesional, industrial, basado en el trabajo, etcétera. En general, esta gama de propuestas surge para distinguirse del doctorado tradicional.

\section{Armonización de la educación doctoral}

En algunos países como Colombia, Brasil, Argentina y Chile no existe distinción entre doctorado profesional y de investigación, como sí sucede con los estudios de maestría. En estos países, con excepción de Brasil, las respectivas agencias de evaluación hacen referencia a un tipo de doctorado académico. En Chile la formación se asocia con la "investigación disciplinar", y el claustro académico debe contar con una "trayectoria académica sobresaliente y pertinente al ámbito disciplinario del programa" (Comisión Nacional de Acreditación (CNA), 2016: 5); en Colombia se señala que el doctorado "acredita la formación y la competencia para el ejercicio académico e investigativo de alta calidad" (Ministerio de Educación Nacional, 2010: 7), y en Argentina: "El Doctorado tiene por objeto la formación de posgraduados que puedan lograr aportes originales en un marco de excelencia académica" (Comisión Nacional de Evaluación y Acreditación Universitaria (CONEAU), 2017: 15).

En el caso de Brasil tampoco se distingue entre un doctorado profesional y uno de investigación, pero se mencionan tres objetivos asociados a diferentes mercados laborales: "Formación posgraduada de docentes para todos los niveles de enseñanza"; "Formación de recursos humanos calificados para el mercado no académico"; y "Fortalecimiento de las bases científica, tecnológica y de innovación" (Coordenação de Aperfeiçoamento de Pessoal de Nível Superior (CAPES), 2014).

En España, con motivo de la conformación del Espacio Europeo de Educación Superior (EEES) y el Espacio Europeo de Investigación - con la participación de la Asociación Europea de Universidades (AEU) como órgano asesor-, se reformó la legislación que regula los programas doctorales para establecer una nueva definición: "El doctorado se entiende como el tercer ciclo de estudios universitarios oficiales, conduce a la adquisición de competencias y habilidades relacionadas con la investigación científica de calidad" (Real Decreto (RD) 99/2011, art. 2.1, en Jiménez, 2017: 127). Esta definición general toma sentido en la descripción de las Escuelas de doctorado, 
entendidas como unidades formadas por una o más instituciones cuyo objetivo primordial es la colaboración con todo tipo de organizaciones dedicadas a actividades de investigación, desarrollo e innovación $(\mathrm{I}+\mathrm{D}+\mathrm{i})$ en el ámbito nacional, con especial interés en el internacional. Estas unidades tienen por objeto la gestión del doctorado en una o más ramas de conocimiento, o en la interdisciplina (RD, 99/2011, art 2.4, en Jiménez, 2017: 127).

El objetivo de la reforma española fue reestructurar los estudios doctorales para evitar que éstos continuaran orientándose hacia la formación del profesor universitario (Jiménez, 2017: 129). No obstante, es importante mencionar que, a pesar de que la legislación española no distingue orientaciones ni modelos doctorales, la Agencia Nacional de Evaluación y Acreditación (ANECA), a través del proceso Verifica, distingue tres ámbitos de pertinencia: científico, académico y social (ANEGA, 2017). Las escuelas de doctorado representan una estructura de formación que también fue identificada en países como Francia, Bélgica, Suiza y Luxemburgo (Paz et al., 2009), por ello se considera que la reforma del doctorado en España representa el ideal de armonización sugerido para la Unión Europea.

En 2010, en Australia, Jim Cumming propuso un modelo doctoral integrado con el cual buscaba dar respuesta a una gran parte del debate actual. $\mathrm{Su}$ propuesta, Integrative model of doctoral enterprise, se compone de dos elementos: doctoral practices y doctoral arrangements. En el primer elemento se encuentran aspectos curriculares, pedagógicos, de investigación y de trabajo. En el segundo se contempla a los académicos, en todos sus niveles; a las comunidades, como el gobierno, la industria, las profesiones y las agencias, y por último a los participantes, es decir, los candidatos, asesores, investigadores, técnicos, pares, etcétera. Dicha estructura se fundamenta en teorías sociológicas de Bourdieu, Giddens y Wenger (Cumming, 2010).

En síntesis, los casos mencionados en este apartado siguen considerando que el doctorado debe basarse en una definición exclusiva, la tradicional o renovada; sin embargo, es importante notar que las agencias de evaluación de Brasil y España ya distinguen tres ámbitos de pertinencia, a pesar de considerar un solo tipo de doctorado.

\section{Diversificación de la educación doctoral}

Contrario a la tendencia anterior, en Europa y China se están identificando diversos tipos de educación doctoral. Paz et al. (2009) señalan tres enfoques: a) de investigador, b) académico y c) profesional. ${ }^{7}$ En el mismo sentido, Bao et al. (2016) identifican nueve modelos: a) de investigación, b) profesional, c) de enseñanza, d) el PhD por trabajo publicado, e) doctorado basado en la práctica, f) el doctorado de nueva ruta, g) interinstitucional, h) de cooperación e i) industrial. En China se identifican algunos doctorados similares a los encontrados en Europa, como los de investigación, profesional, interinstitucional e industrial, y adicionalmente, el integrado (Bao et al., 2016). ${ }^{8}$

A partir del recuento anterior de los tipos de educación doctoral, interesa destacar la existencia de una diversidad de características y objetivos inmersos en los doctorados. En este artículo solo se discuten seis de estos tipos por ser los que se asocian a distintas etapas de desarrollo del doctorado de investigación y del profesional.

\footnotetext{
${ }^{7}$ De acuerdo con los autores, entre los 44 países que conforman el EEES, 91.1\% cuenta con el enfoque investigador, 35.5\% con el enfoque académico y $24.4 \%$ con el profesional. Estos datos son poco claros, pues los autores omiten la definición de cada enfoque y la forma en la que calcularon dichos porcentajes. Aun así, es importante considerarlos, pues de acuerdo con la revisión de la literatura, el enfoque de investigador podría referirse a las escuelas doctorales, el académico al doctorado tradicional de investigación, y el profesional al que se define en este artículo.

${ }^{8}$ Bao et al. (2016) y Paz et al. (2009) utilizan el término enfoque y modelo para referirse a los doctorados, sin embargo, en el segundo caso, posiblemente algunos de los doctorados mencionados se refieren a modalidades de titulación más que a modelos. Por lo anterior, en este artículo se consideró más adecuado utilizar el término "tipos de educación doctoral”, por ser más general y "orientación”, por ser el término utilizado en el PNPC para distinguir entre el doctorado de investigación y el profesional.
} 
El primero es el doctorado de investigación, asociado a las identidades académicas de la universidad alemana del siglo XIX, donde los estudiantes eran considerados aprendices dirigidos por un doctor hacia el trabajo de la investigación. Este doctorado fue adaptado en diversos países con gran éxito, como en el caso de Estados Unidos (Arimoto, en Teichler, 2015; Bao et al., 2016), no obstante, ha sido fuertemente cuestionado, situación que ha generado distintas reformas, como la del caso español. Una de las críticas principales es que el doctorado de investigación forma al investigador como "guardián de una disciplina", y su desempeño laboral se centra en las actividades académicas (Bao et al., 2016).

El segundo es el doctorado basado en la enseñanza, se denomina así porque la mayoría del trabajo se realiza mediante cursos, y cuenta con un plan de estudios fijo. Si bien exige una contribución original al conocimiento, en lugar de hacerlo mediante tesis se presenta un informe de proyecto que se califica a través de un examen oral. Este informe es equivalente a una disertación. La oferta de este tipo de doctorados se puede encontrar en el Reino Unido (Bao et al., 2016).

El tercero es el doctorado profesional, cuyas características fueron descritas por Bourner et al. (2001) en Inglaterra, a través de la identificación y análisis de 128 programas en educación, medicina, psicología clínica, administración de negocios, ingeniería, psicología, artes musicales, arquitectura, ciencia veterinaria, ciencia dental, salud pública, psicología ocupacional, psicoterapia clínica, psicoterapia psicoanalítica, teología, artes, farmacia, finanzas, trabajo social, psicología del adolescente y humanidades.

Bourner et al. (2001) describen al doctorado profesional comparándolo con el $\mathrm{PhD}$; así identifican 19 aspectos que los distinguen, pero el aspecto más destacado de esta comparación es que el estudiante del doctorado profesional busca mejorar su práctica, y se interesa por la investigación en la medida en que ésta ofrece soluciones a problemas específicos de su profesión; en cambio, el estudiante del doctorado de investigación busca convertirse en investigador y hacer carrera académica.

En síntesis, se considera que el objetivo principal del doctorado profesional es "preparar a los egresados para la resolución de los problemas difíciles en algún campo profesional, así como la generación de conocimientos aplicados. Este doctorado con frecuencia se completa estudiando medio tiempo, al lado de las responsabilidades laborales profesionales" (Teichler, 2015: 33).

La revisión de los estudios más recientes respecto al doctorado profesional permitió identificar tres generaciones de desarrollo. De acuerdo con Maxwell (2003), Costley y Lester (2012), el doctorado profesional de primera generación se caracterizó por el apego a la estructura académica de las universidades (Maxwell, 2003). La transición de la primera a la segunda generación de doctorados profesionales se identificó en aquellos programas que no sólo se interesaron por la generación del conocimiento especializado, sino también por su aplicación; éstos centraron el aprendizaje no sólo en los cursos y en la supervisión individual, sino en la asociación entre la universidad y los empleadores. Este doctorado coincide con el currículo híbrido (Lee, en Maxwell, 2003), en el que interactúan elementos de tres esferas de conocimiento: la universidad, la profesión y el trabajo. Esta característica coincide con los doctorados profesionales que describen Bouner et al. (2001) y Taylor (2004) en Inglaterra, así como Schildkraut y Stafford (2015) en EUA.

La tercera generación del doctorado profesional está representada por el doctorado basado en el trabajo (work-based doctorate). Este doctorado deja a un lado la profesión para centrarse en el aprendizaje basado en las necesidades de la práctica laboral; así, el currículo está determinado por el trabajo, y se caracteriza por ser multidisciplinar. Los autores asocian a este doctorado con principios filosóficos y pedagógicos de Jhon Dewey, D. A. Schön y Reg Revan, entre otros (Costley y Lester, 2012). 
El último es el doctorado industrial, identificado también como doctorado en Ingeniería (EngD). El interés principal, en este caso, es la formación de investigadores cuyo proyecto de investigación se refiera a problemas de la industria. La evaluación se basa en una cartera de proyectos de investigación, no en una tesis; y la supervisión de los doctorandos suele apoyarse en personal de la industria y personal académico. Estos doctorados se pueden encontrar en Inglaterra (Taylor, 2004), Alemania, Bélgica (De Grande et al., 2014), China (Bao et al., 2016) y Australia (Manathunga et al., 2012). Es importante señalar que algunos autores consideran que los doctorados de ingeniería son también doctorados profesionales (Bao et al., 2016; Taylor, 2004), sin embargo, el tema no ha sido discutido en la literatura.

De los seis doctorados identificados, tres de ellos, el industrial, el profesional de segunda generación y el doctorado basado en el trabajo tienen un elemento común: el aprendizaje basado en la investigación para la aplicación de conocimientos. Los otros tres, el de investigación, el profesional de primera generación y el de enseñanza tienen en común la presentación de una tesis o trabajo terminal equivalente a la disertación, por lo que se consideran opciones más orientadas a las tradiciones académicas.

Las dos tendencias identificadas en la revisión de literatura muestran dos formas de enfrentar una multiplicidad de exigencias para impactar en el desarrollo de las profesiones, la academia, la industria y otras necesidades sociales; de la misma manera, estas tendencias también permiten comprender las maneras en las que se están organizando los instrumentos de evaluación, un ejemplo de ello es el PNPG.

\section{El doctorado profesional y de investigación en México a través de sus instrumentos de evaluación}

La evaluación del doctorado en México se impulsó desde 1991 a través de los programas antecesores del PNPC, pero desde ese año hasta 2011, sólo existía el doctorado con orientación a la investigación en la modalidad escolarizada. En 2012 se incorporó la modalidad con la industria, ${ }^{9}$ en 2014 la orientación profesional, y en 2016 la modalidad no escolarizada, aunque ésta sólo aplica para los doctorados profesionales. ${ }^{10} \mathrm{De}$ esta manera, el doctorado de investigación únicamente se puede evaluar en la modalidad escolarizada y con la industria; en cambio, el doctorado profesional puede evaluarse en las tres modalidades.

En 2018, el padrón del PNPC registró la calidad de 643 programas de doctorado en toda la República mexicana; de ellos, 631 se orientan a la investigación en la modalidad escolarizada, y otros cinco en la modalidad con la industria; siete doctorados con orientación profesional, cinco en la modalidad escolarizada, uno con la industria y uno en la no escolarizada (CONACyT, 2018). En total, se cuentan 12 doctorados distintos a los tradicionales de investigación (ver cuadro 1). De acuerdo con esta clasificación, tanto el doctorado de investigación como el profesional se pueden ofrecer en la modalidad con la industria, por lo que podrían considerarse equivalentes al doctorado industrial reportado en la literatura.

De acuerdo con el PNPG la calidad de un programa de posgrado es una propiedad que se adquiere una vez cumplidos los criterios establecidos en sus Marcos de Referencia (CONACyT, 2011). Los programas pueden alcanzar distintos niveles de competencia: reciente creación, en desarrollo, consolidado y de competencia internacional, de acuerdo con el grado de cumplimiento de los criterios.

\footnotetext{
${ }^{9}$ Aplica para los tres niveles de posgrado.

${ }^{10}$ Esta orientación existía tiempo atrás, sin embargo, no aplicaba para el nivel de doctorado.
} 
Cuadro 1. Doctorados profesionales y con la industria registrados en el PNPC 2018

\begin{tabular}{|c|c|c|c|c|}
\hline Orientación & Número & Nombre del doctorado & Institución & Modalidad \\
\hline \multirow{7}{*}{ Profesional } & 1 & $\begin{array}{l}\text { Innovación en tecnología } \\
\text { educativa }\end{array}$ & Universidad Autónoma de Querétaro & No escolarizado \\
\hline & 2 & $\begin{array}{l}\text { En estudios económico- } \\
\text { administrativos }\end{array}$ & Universidad Juárez Autónoma de Tabasco & Escolarizado \\
\hline & 3 & Ingeniería Mecatrónica & Universidad Popular Autónoma del Estado de Puebla, A. C. & Con la industria \\
\hline & 4 & Internacional en Turismo & Universidad Anáhuac, México Norte & Escolarizado \\
\hline & 5 & $\begin{array}{l}\text { Defensa y seguridad } \\
\text { nacional }\end{array}$ & Secretaría de la Marina/Universidad Naval & Escolarizado \\
\hline & 6 & $\begin{array}{l}\text { Administración y alta } \\
\text { dirección }\end{array}$ & Universidad Autónoma de Coahuila & Escolarizado \\
\hline & 7 & Contaduría & Universidad Autónoma de Nuevo León & Escolarizado \\
\hline \multirow{5}{*}{ Investigación } & 8 & $\begin{array}{l}\text { Ingeniería y tecnología } \\
\text { aplicada }\end{array}$ & Universidad Autónoma de Zacatecas & Con la industria \\
\hline & 9 & Manufactura avanzada & $\begin{array}{l}\text { CIATEQ, A.C. de San Luis Potosí, Hidalgo, Aguascalientes, } \\
\text { Estado de México, Jalisco y Corporativo Querétaro }\end{array}$ & Con la industria \\
\hline & 10 & Ciencias de la ingeniería & Universidad Iberoamericana, A. C. & Con la industria \\
\hline & 11 & $\begin{array}{l}\text { Biotecnología } \\
\text { reproductiva }\end{array}$ & Instituto Politécnico Nacional & Con la industria \\
\hline & 12 & Ciencias de la ingeniería & $\begin{array}{l}\text { Instituto Tecnológico y de Estudios Superiores de } \\
\text { Occidente }\end{array}$ & Con la industria \\
\hline
\end{tabular}

Fuente: elaboración propia con base en el Padrón del PNPC 2018

* Probablemente en el PNPC existen más doctorados que podrían asumirse como profesionales, pero no lo hicieron porque no existía dicha clasificación. 


\section{Principales rasgos del doctorado de investigación y profesional ${ }^{11}$}

De acuerdo con los documentos del PNPC, el doctorado de investigación se caracteriza por la formación para la investigación interdisciplinaria, en un entorno internacional para adquirir competencias transferibles e innovación; así como la capacidad crítica y creativa a través de investigaciones originales. En cambio, el doctorado profesional busca la formación para mejorar el ejercicio profesional u ocupacional y la adquisición de conocimientos a través de la práctica en un laboratorio o en el propio trabajo.

Respecto a las disciplinas o campos de conocimiento, para la orientación a la investigación no se expresa alguna preferencia, sin embargo, algunos estudios señalan que en el doctorado tradicional de investigación había una preferencia por campos disciplinarios como la física, biología, matemáticas, sociología, filosofía, historia, etcétera (Arredondo et al., 2006: 16); en cambio, para la orientación profesional se especifican algunas disciplinas asociadas a las profesiones liberales (cuadro 2). Además de la preferencia por ciertos campos de conocimiento, se criticaba al PNPC por permitir que, a través de los criterios de evaluación se descalificara la orientación tecnológica (ANUIES, en Díaz-Barriga, 2009), y se desconociera la importancia de la formación profesional, sugiriendo que la única referencia de calidad estaba constituida por los programas de investigación (Díaz-Barriga, 2009).

Una de las diferencias más importantes entre el doctorado de investigación y el profesional es la relativa al problema de investigación sobre el que se desarrollan las tesis de los estudiantes. Mientras que en el doctorado profesional se busca la solución de un problema del usuario; en el de investigación se busca la solución original a un problema de investigación, esperando conocimientos que den lugar a publicaciones (CONAGyT, 2015a: 15). No obstante, la redacción del último Marco de referencia hace evidente una dificultad importante en este criterio para mostrar las diferencias entre una orientación y otra. El uso del término "investigación" resulta ambiguo después de utilizar el término "investigación aplicada u orientada", de manera que, en algunos apartados, el lector podría interpretar que el término "investigación" se refiere a "investigación básica".

\section{Cambios en la definición del doctorado}

Antes de que en el PNPG se propusieran distintas orientaciones y modalidades, estaba sobreentendido que el doctorado tenía por objetivo la formación de académicos (Díaz-Barriga, 2009; Patiño, 2018), pero a partir de 2012, con la incorporación de los posgrados con la industria, se empiezan a realizar cambios de manera paulatina en diversos documentos del CONACyT. En 2013, la definición del doctorado ya no incluye la formación de docentes universitarios, e incorpora la formación para la investigación, desarrollo tecnológico e innovación (Informe general del estado de la ciencia, la tecnología y la innovación 2013, en Patiño, 2018). Posteriormente, en el Marco de Referencia 2015, versión 6, se identifican nuevos elementos en la definición de los doctorados de investigación, como las competencias transferibles, el entorno interdisciplinario y la cooperación internacional, por lo que se infiere que la definición del doctorado no sólo se transformó para incluir al doctorado profesional sino también para renovar la definición del doctorado de investigación.

\section{El doctorado profesional y de investigación a la luz de la política científica en México}

Los cambios en la definición de un doctorado y la incorporación del doctorado profesional al PNPG coinciden con el papel central que se ha brindado a la innovación e investigación aplicada en los documentos de política pública a partir del Programa Especial de Ciencia y Tecnología (PECyT, 2008-2012) y el

\footnotetext{
${ }^{11}$ En este artículo se analizan los elementos que componen las dos orientaciones doctorales sin considerar la modalidad con la industria y no escolarizada.
} 


\section{Cuadro 2. Rasgos de los doctorados de investigación y profesional}

\begin{tabular}{|c|c|c|}
\hline Categorías & Investigación & Profesional \\
\hline Objetivos específicos & $\begin{array}{l}\text { a) brindar "adiestramiento metódico en investigación en un } \\
\text { entorno interdisciplinario, cooperativo e internacional"; } \\
\text { b) ofrecer una "formación amplia y sólida en un campo de } \\
\text { conocimiento con una alta capacidad crítica y creativa a través } \\
\text { de investigaciones originales"; } \\
\text { c) brindar "formación en competencias transferibles, } \\
\text { fomentando el contacto entre doctorandos de distintas } \\
\text { disciplinas, la creatividad y la innovación" (CONACyT, 2015a: 12). }\end{array}$ & $\begin{array}{l}\text { a) profundizar y ampliar conocimientos y destrezas para mejorar } \\
\text { el ejercicio profesional; } \\
\text { b) adquirir conocimientos específicos a través de prácticas de } \\
\text { laboratorio o prácticas profesionales aplicadas al trabajo; } \\
\text { c) adiestrar para solucionar problemas de una ocupación } \\
\text { específica; } \\
\text { d) impartir conferencias y seminarios como una actividad } \\
\text { complementaria; } \\
\text { e) trabajar sobre líneas de investigación vinculadas a la actividad } \\
\text { profesional. }\end{array}$ \\
\hline $\begin{array}{l}\text { Disciplinas o campos de } \\
\text { conocimiento }\end{array}$ & No se especifican disciplinas o campos de conocimiento. & $\begin{array}{l}\text { Estos programas pueden basarse en una profesión o ser más } \\
\text { genéricos. Los campos mencionados son: educación, derecho, } \\
\text { ciencias de la salud, medicina clínica, administración, psicología } \\
\text { clínica, psicología educacional, enfermería, psicoterapia y } \\
\text { finanzas. }\end{array}$ \\
\hline Estructura curricular & $\begin{array}{l}\text { Se conforma por seminarios y culmina con la realización y } \\
\text { defensa de una tesis que brinde una solución original a un } \\
\text { problema de investigación. } \\
\text { Además de ello, la formación se apoya en conferencias y talleres. }\end{array}$ & $\begin{array}{l}\text { Suele contener cursos, proyectos de investigación orientados al } \\
\text { lugar de trabajo y prácticas profesionales. Los contenidos que se } \\
\text { revisan en los cursos suelen ser de "naturaleza aplicada" y tienen } \\
\text { relación con el trabajo. } \\
\text { Estos programas también culminan con la defensa de una } \\
\text { tesis enfocada en algún problema del usuario (profesión u } \\
\text { ocupación). }\end{array}$ \\
\hline
\end{tabular}

Fuente: elaboración propia con base en el Marco de referencia para la evaluación y seguimiento de posgrados presenciales y Anexos A (cONACyT, 2015a; 2015b; 2015c).

PECiTI (2014-2018). En estos documentos no se hace referencia a las orientaciones doctorales del PNPC, pero se expresa que este nivel educativo forma a los investigadores que generarán investigación, desarrollo tecnológico e innovación, con el fin de mejorar productos y servicios para la sociedad a través de su inserción laboral en "instituciones, gobierno, empresas y otros organismos" (CONAGyT, en Patiño, 2018: 6).

De acuerdo con el Marco de referencia del PNPG, el doctorado profesional tiene cuatro funciones: a) apoyar el desarrollo de investigación en disciplinas emergentes, b) consolidar vínculos con la sociedad, c) brindar nuevas oportunidades para los estudiantes y d) brindar una solución a la preocupación que existe en las empresas sobre la falta de experiencia práctica y habilidades para el trabajo de los estudiantes de los posgrados de investigación (CONACyT en Patiño, 2018).

\section{Diferentes criterios de evaluación en el PNPC}

Con la incorporación del doctorado profesional al PNPC, se hizo una adaptación de siete criterios, de un total de 15 que se utilizaban para evaluar al doctorado de investigación (cuadro 3).

Las principales diferencias entre la evaluación de un doctorado de investigación y uno profesional se sintetizan en los siguientes aspectos:

a) Para el doctorado profesional se hace mayor énfasis que para el de investigación en la vinculación con el sector productivo, este aspecto está contemplado desde el plan de estudios y la movilidad, hasta el mercado laboral de los graduados.

b) Para los doctorados de investigación se sigue exigiendo la mayoría de estudiantes con dedicación de tiempo completo, en cambio para los profesionales, 


\section{Cuadro 3. Criterios en los que difieren la orientación profesional y la de investigación}

\begin{tabular}{|c|c|c|}
\hline Categorías & Criterios & $\begin{array}{c}\text { Diferencia investigación/ } \\
\text { profesional }\end{array}$ \\
\hline \multirow{4}{*}{$\begin{array}{l}\text { 1. Estructura y personal académico } \\
\text { del programa }\end{array}$} & 1. Plan de estudios & $x$ \\
\hline & 2. Proceso de enseñanza aprendizaje & \\
\hline & 3. Núcleo Académico Básico & $x$ \\
\hline & 4. Líneas de generación y/o aplicación del conocimiento & \\
\hline \multirow{4}{*}{ 2. Estudiantes } & 5. Ingreso de estudiantes & \\
\hline & 6. Seguimiento de la trayectoria académica de los estudiantes & \\
\hline & 7. Movilidad de los estudiantes & $x$ \\
\hline & 8. Dedicación de los estudiantes al programa & $x$ \\
\hline \multirow{2}{*}{ 3. Infraestructura del programa } & 9. Espacios, laboratorios, talleres y equipamiento & \\
\hline & 10. Biblioteca y tecnologías de información y comunicación & \\
\hline \multirow{5}{*}{ 4. Resultados y vinculación } & 11. Trascendencia, cobertura y evolución del programa & $x$ \\
\hline & 12. Efectividad del posgrado & \\
\hline & 13. Contribución al conocimiento & $x$ \\
\hline & 14. Vinculación & $x$ \\
\hline & 15. Financiamiento & \\
\hline
\end{tabular}

Fuente: elaboración propia con base en el Marco de referencia para la evaluación y seguimiento de programas de posgrado presenciales, versión 6 (conAcyt, $2015 a$ ) y los Anexos de cada orientación. 
pueden registrarse programas de tiempo parcial, sin derecho a solicitar beca de CONACyT para sus estudiantes debido a que se espera que muchos de ellos continúen con una relación laboral y sus tesis aborden algún problema de su ámbito de trabajo. c) Los profesores que integran los Núcleos Académicos Básicos (NAB) de los programas de investigación deben contar con un porcentaje adecuado de miembros con reconocimiento del Sistema Nacional de Investigadores (SNI); en cambio los profesores de los doctorados profesionales deben contar con el SNI o con una productividad equivalente, relacionada con su desempeño profesional. ${ }^{12}$ Cabe destacar que la membrecía del SNI para los miembros del NAB ha sido uno de los indicadores más difíciles y polémicos, dado que este reconocimiento se otorga a los académicos que logran cumplir determinado número de publicaciones en revistas de alto nivel. Al modificar este criterio se está aceptando que el SNI no es el indicador adecuado para valorar la trayectoria de otro tipo de profesores de campos de conocimiento profesionales.

d) Otra diferencia importante se encuentra en el tipo de problemas de investigación que se atiende en cada programa, para los profesionales está muy claro, son de tipo aplicado; en cambio para los de investigación podría existir una disyuntiva entre los conocimientos publicables y el desarrollo tecnológico y la innovación.

e) La pertinencia del programa se evalúa en gran medida por el sector laboral en el que se desempeñan los graduados, así, se espera que los egresados de los doctorados de investigación logren el reconocimiento del SNI, para lo cual tendrían que laborar en alguna institución que se dedique a la investigación, mientras que para los doctorados profesionales se espera la inserción laboral en el sector productivo.
Es importante mencionar que en los documentos referidos se aprecia dificultad para definir con claridad cuál es el indicador adecuado para evaluar la pertinencia y la contribución al conocimiento de cada orientación, pero con este estudio no se logra distinguir si esta ambigüedad se debe al deseo de establecer criterios flexibles o a la falta de claridad sobre la distinción entre las orientaciones. Para finalizar, es necesario mencionar que los cambios en el PNPG siguen en marcha. Al inicio de 2018, la Dirección de Posgrados del CONACyT coordinó la revisión de la nueva versión del PNPC, es decir, la versión 7, en la cual la pertinencia adquiere un valor fundamental.

\section{Conclusiones}

El objetivo de este artículo fue identificar bajo qué criterios se justifica el doctorado profesional en comparación con el de investigación. Como se pudo observar en los resultados, el doctorado profesional deriva de un enfoque que valora el aprendizaje tanto en la academia como en el trabajo; que resalta la importancia de la investigación aplicada para la mejora de la profesión u ocupación; y que busca la vinculación de sus graduados con el sector productivo.

En el caso del PNPC, la comparación entre el doctorado de investigación y el profesional permite abrir la discusión sobre cuáles serían los referentes más adecuados para cada orientación. La ambigüedad en algunos criterios de evaluación evidencia la necesidad de seguir discutiendo cuáles son las aportaciones de cada modalidad. Por ejemplo, es necesario preguntarse si la investigación publicable y la investigación aplicada son los referentes adecuados. El reto no sólo es hacer esfuerzos por diseñar nuevos referentes coherentes con el doctorado profesional, también es necesario definir con claridad qué se espera del doctorado de investigación a partir de los cambios discutidos aquí.

\footnotetext{
${ }^{12}$ Los porcentajes adecuados para cada programa están establecidos en el Anexo A, de acuerdo con el nivel de competencia.
} 
La identificación de dos tendencias sobre la definición de la educación doctoral permite mostrar que este nivel de estudios atraviesa una etapa de transformación global, impulsada, en gran medida, por el deseo de reorientar el destino laboral de los graduados y, por ende, la diversificación de sectores beneficiarios. Hasta este punto hay consenso, lo que no se ha discutido aún es la mejor forma para impulsar estos cambios. Hasta el momento se ha pensado que existen dos opciones: reformar el doctorado tradicional o proponer nuevos. Se podría pensar que la mejor opción es crear un doctorado integral, sin embargo, parece que esta concepción dificulta el establecimiento de criterios de evaluación y de referentes pues, como se observa en el caso de Brasil, España y México, en algún momento se establecen diferentes ámbitos de pertinencia.

La discusión anterior pone sobre la mesa la preocupación por la pertinencia de los estudios doctorales, no obstante, es necesario hacer mayores esfuerzos para definir qué significa este concepto y cómo se establece en la práctica evaluadora, es necesario seguir discutiendo las diferencias entre los ámbitos científicos, académicos, profesionales y sociales cacaso el académico y el profesional no se forman como científicos también?, y ¿no son todos ámbitos sociales? La discusión anterior es indispensable porque se requieren estudios de pertinencia que orienten la toma de decisiones para la apertura de nuevos doctorados y para la actualización o reestructura de los que ya existen. Por ejemplo, ¿cómo saber qué tipo de doctorado se requiere y en qué campo de conocimiento es adecuado? Valdría la pena partir de la pregunta ¿qué tipo de problemas sociales atenderá el nuevo doctorado y qué se requiere para ello?

Es importante reconocer que las autoridades del PNPC optaron por la diversificación de tipos doctorales con base en nuevos referentes de evaluación, en un esfuerzo por atender nuevos sectores, pero la tarea no está concluida, habrá que poner a prueba las nuevas categorías para seguir haciendo ajustes. 


\section{Referencias}

Agencia Nacional de Evaluación y Acreditación (ANECA) (2017), "Plantilla de evaluación para la verificación de enseñanzas oficiales de doctorado", Madrid, <http://www.aneca.es/Programas-de-evaluacion/ VERIFICA/Verificacion-de-Ensenanzas-deDoctorado/Documentos-y-herramientas $>$ [Consulta: diciembre de 2017].

Arredondo Galván, V. M., G. Pérez Rivera y P. Morán Oviedo (2006), "Políticas de posgrado", en Reencuentro, núm. 45, pp. 1-24.

Auriol, L. (2010), Careers of doctorate holders: Employment and mobility patterns, París, OECD Publishing (OECD Science, Technology and Industry Working Papers), <https:// doi.org/10.1787/5kmh8phxvvf5-en> [Consulta: julio de 2010].

Bao, Y., B. M. Kehm y Y. Ma (2016), "From product to process. The reform of doctoral education in Europe and China", en Studies in Higher Education, vol. 43, núm. 3, pp. 524-541.

Bonilla Marín, M. (2015), "Cuarta parte: los actores de posgrado", en Marcial Bonilla (coord.), Diagnóstico del posgrado en México: Nacional, Torreón, México, Comepo, A. C., pp. 80-110, <http://www.comepo.org.mx/ diagnostico/> [Consulta: marzo de 2016].

Bourner, T., R. Bowden y L. Stuart (2001), "Professional doctorates in England", en Studies in Higher Education, vol. 26, núm. 1, pp. 65-83.

Coordenação de Aperfeiçoamento de Pessoal de Nível Superior (CAPES) (2014), Objetivos do Sistema Nacional de Pós-Graduação (Sobre a Avaliação de la CAPES), Brasil, <http://capes.gov.br/avaliacao/sobre-a-avaliacao> [Consulta: noviembre de 2017].

Comisión Nacional de Acreditación (GNA) (2016), "Operacionalización de criterios de evaluación para la acreditación de programas de postgrado: doctorado, magíster académico y magíster profesional", Santiago de Chile, <https://www.cnachile.cl/Documentos $\% 20$ de $\% 20$ Paginas / Operacionalizaci $\% \mathrm{C} 3 \% \mathrm{~B} 3 \mathrm{n} \% 20$ de $\% 20$ criterios $\% 20 \mathrm{de} \% 20$ Postgrado.pdf> [Consulta: noviembre de 2017].

Comisión Nacional de Evaluación y Acreditación Universitaria (CONEAU) (2017), "Introducción", en Posgrados acreditados de la República Argentina, Buenos Aires, <http://www.coneau.gov.ar/archivos/publicaciones/ documentos/CatalogoPosgrados2017.pdf> [Consulta: noviembre de 2017].

Consejo Nacional de Ciencia y Tecnología (CONACyT (2011), "Glosario de términos del PNPG para Programas de Posgrado Escolarizados, versión 2", México, CONACyT /SEP-Subsecretaría de Educación Superior-Dirección Adjunta de Posgrado y Becas, <http://dsia.uv.mx/sipo/Material_apoyo/Glosario_ Escolarizada.pdf $>$ [Consulta: mayo de 2016].

CONAGyT (2015a), Marco de referencia para la evaluación y seguimiento de programas de posgrado presenciales [versión 6], México, CONACyT/SEP-Subsecretaría de Educación Superior, <https://www.conacyt.gob.mx/index.php/ becas-y-posgrados/programa-nacional-de-posgradosde-calidad/convocatorias-avisos-y-resultados / convocatorias-cerradas-pnpc/9005-marco-dereferencia-modalidad-escolarizada/file $>$ [Consulta: mayo de 2016].

CONACyT (2015b), Anexo A: Programas de orientación a la investigación [versión 4.1], México, CONACyT/ SEP-Subsecretaría de Educación Superior, <https:// www.conacyt.gob.mx/index.php/becas-y-posgrados/ programa-nacional-de-posgrados-de-calidad/ convocatorias-avisos-y-resultados/resultadospnpc/9040-anexo-a-orientacion-investigacion/file> [Consulta: junio de 2017].

CONACyT (2015c), Anexo A: Programas de orientación profesional [versión 4.1. PDF no disponible en línea], México, GONAGyT/SEP-Subsecretaría de Educación Superior.

CONACyT (2018), Padrón PNPC [Sistema de consultas], México, <http://svrtmp.main.conacyt.mx/ ConsultasPNPC/listar_padron.php> [Consulta: enero de 2018]. 
Costely, C. y S. Lester (2012), "Work-based doctorates: Professional extension at the highest levels", en Studies in Higher Education, vol. 37, núm. 3, pp. 257-269.

Cumming, J. (2010), "Doctoral enterprise: a holistic conception of evolving practices and arrangements", en Studies in Higher Education, núm. 35, vol. 1, pp. 25-39.

De Grande, H., K. De Boyser, K. Vandevelde y R. Van Rosem (2014), "From academia to industry: are doctorate holders ready?", en Fournal of the Knowledge Economy, vol. 5, núm. 3, pp. 538-561.

Díaz-Barriga, Ángel (2009), “Criterios de evaluación externa de los posgrados en México. Un sistema de acreditación que desconoce su pertinencia social", en Teresa Pacheco Méndez y Ángel Díaz-Barriga (coords.), El posgrado en educación en México, México, UNAM-Coordinación de Humanidades/Instituto de Investigaciones sobre la Universidad y la Educación, pp. 45-87.

Jiménez-Ramírez, M. (2017), "Los nuevos estudios de doctorado en España: avances y retos para su convergencia con Europa", en Revista Iberoamericana de Educación Superior, vol. VIII, núm. 21, pp. 123-137.

Manathunga, C., R. Pitt, L. Cox, P. Boreham, G. Mellick y P. Lant (2012), "Evaluating industry-based doctoral research programs: perspectives and outcomes of Australian Cooperative Research Centre Graduates", en Studies in Higher Education, vol. 37, núm. 7, pp. 843858.

Maxwell, T. (2003), "From first to second generation professional doctorate", en Studies in Higher Education, vol. 28, núm. 3, pp. 279-291.

Ministerio de Educación Nacional (2010), "Lineamientos para la acreditación de alta calidad de programas de maestría y doctorado", Colombia, Consejo Nacional de Acreditación (CNA), <https://www.cna.gov.co/1741/ articles-186359_Lineamiento_Maestria_Doctorados. pdf> [Consulta: noviembre de 2017].

Mollis, M. (2010), "Imágenes de posgrados: entre la academia, el mercado y la integración regional", en M. Mollis, J. Núñez Jover y C. García Guadilla, Políticas de posgrado y conocimiento público en América Latina y el Caribe,
Buenos Aires, CLACsO, pp. 13-56.

Morles, V. y J. R. León (2002), "Los estudios de posgrado en Iberoamérica y el Caribe: evolución y tendencias", en J. Castro Lamas y J. Núñez Jover (coords.), Gestión de la calidad del postgrado en Iberoamérica. Experiencias nacionales, Salamanca, España, AUIP, pp. 18-35.

Nohlen, D. (2006), "Método comparativo", en Diccionario de ciencia política: teorías, métodos, conceptos (dos tomos), pp. 1-8.

Núñez Jover, J. (2002), “Introducción”, en J. Castro Lamas y J. Núñez Jover (coords.), Gestión de la calidad del postgrado en Iberoamérica. Experiencias nacionales, Salamanca, España, AUIP, pp. 1-17.

PatiñoSalceda,Josefina(2017), "Doctoradosprofesionales: concepciones y debate. Entrevista a Luis Ponce Ramírez", en Revista Electrónica de Investigación Educativa, vol. 19, núm. 4, pp. 1-15.

Patiño Salceda, Josefina (2018), "Formar para investigar y profesionalizar para innovar" [manuscrito inédito], México, Universidad Nacional Autónoma de MéxicoInstituto de Investigaciones sobre la Universidad y la Educación.

Paz Bermúdez, M., Á. Castro, J. C. Sierra y G. BuelaCasal (2009), "Análisis descriptivo transnacional de los estudios de doctorado en el EEES", Revista Psicodidáctica, vol. 14, núm. 2, pp. 193-210.

Schildkraut, J. y M. Stafford (2015), "Researching professionals or professional researchers? A comparison of professional doctorate and $\mathrm{PhD}$ Programs in Criminology \& Criminal Justice", en American fournal and Criminal fustice, vol. 40, núm. 1, pp. 183-198.

Taylor, J. (2004), "The United Kindom”, en J. Sadlak (ed.), Doctoral studies and qualifications in Europe and The United States: status and prospects, Bucharest, UNESCO, pp. 231258.

Teichler, U. (2015), "Educación y formación doctoral: una mirada por países y disciplinas", en M. de Ibarrola y L. W. Anderson (coord.), La formación de nuevos investigadores educativos. Diálogos y debates, México, ANUIES, pp. 19-55.

Valenzuela Morales, Clelia (2014), "Evaluación de programas de doctorado transnacionales: Una 
experiencia en Chile", en Revista Iberoamericana de Educación Superior, vol. V, núm. 12, pp. 70-86.

Wildy, H., S. Peden y K. Chan (2015), The rise of professional doctorates: case studies of the Doctorate in Education in China, Iceland and Australia, en Studies in Higher Education, vol. 40, núm. 5, pp. 761-774.

\section{Cómo citar este artículo:}

Patiño Salceda, Josefina (2019), "Análisis comparativo entre el doctorado profesional y de investigación en México", en Revista Iberoamericana de Educación Superior (RIES), México, UNAM-IISUE/Universia, vol. X, Núm. 28, pp. 25-41, DoI: https://doi.org/10.22201/ iisue.20072872e.2019.28.427 [consulta: fecha de última consulta]. 